\title{
Measuring out the relation between formal and conceptual semantics
}

\section{Tillmann Pross \& Antje Roßdeutscher}

In this paper, we argue that contemporary approaches of constructionalist syntax in which there is no generative lexicon provide an interface between formal and conceptual semantics with which the gap between formal and conceptual semantics can be bridged. We introduce the framework with the discussion of formal and conceptual aspects of meaning in German spatial denominal prefix- and particle verbs. We then show the representation of both formal and conceptual semantics in the same framework that allows to measure out the relation between formal and conceptual semantics in terms of the distribution of direct objects over verbs and corroborate our proposal with a corpus study.

\section{Introduction}

In this paper, we perceive the 'gap' between formal and conceptual semantics as pertaining to the different principles according to which the formal semantics of sentences and the conceptual semantics of lexical items is derived. On the one hand, the formal semantics of a sentence is determined compositionally from the meanings of the constituents of the sentence according to the syntactic analysis of the sentence. On the other, the meaning of a word is determined by the arrangement of elements from a fixed set of basic concepts in a lexical entry where the arrangement is not governed by syntactic structures similar to that of sentences.

This work was supported by a DFG grant to the project B4 'Lexikalische Information und ihre Entfaltung im Kontext von Wortbildung, Satz und Diskurs', as part of the Collaborative Research Center 732 Incremental Specification in Context at the University of Stuttgart. 
In order to bridge the gap between formal and conceptual semantics, we propose to make use of a logical form framework in which the perceived gap between formal and conceptual semantics does not manifest itself in a difference of the derivation of meaning in words and sentences. Instead, in the proposed framework, word meaning, and in particular the meaning of morphologically complex words, is structured according to the same syntactic principles underlying the structure of sentence meaning. Our approach is introduced with the discussion of spatial German denominal prefix- and particle-verbs (henceforth short 'p-verbs') as in (1).

(1) abstützen (to support), aufbahren (to lay sb. out), aufbocken (to jack up), aufkanten (to tilt sth.), aufstocken (to ramp up), einlagern (to put in a store) einsacken (to bag sth.), einkellern (to store), einkerkern (to incarcerate), einsperren (to cage), überbrücken (to bridge), überdecken (to cover), überdeckeln (to cover with a lid), überpflastern (to cobble), ummauern (to wall), umzäunen (to fence in), unterfüttern (to reline), untermauern (to support), untertunneln (to tunnel under), verstreben (to strut)

Based on a detailed analysis of the p-verbs in (2) at the syntax-semantics interface, we show how in our approach the formal components of word meaning can be separated from the conceptual components of word meaning.

(2) a. eine Terrasse überdachen

a terrace over.prfx.roof

to roof a terrace

b. einen Dachstuhl abstützen

a truss up.prtc.stilt

to prop up a truss

c. eine Flasche in den Keller einlagern

a bottle in the cellar in.prtc.store

to put a bottle in the cellar

Furthermore, we argue that the separation of formal and conceptual meaning in a word allows to correlate the relation between formal and conceptual meaning in a p-verb with the restrictions on fillers of argument slots imposed by the p-verb. More specifically, we propose that the relation between formal and conceptual meaning in a given p-verb can be measured out in terms of the distribution of possible fillers of argument slots over $\mathrm{p}$-verbs which in turn provides a linguistic 
characterization of conceptual meaning independent of assumptions about the cognitive structures underlying conceptual meaning.

The paper is structured as follows. In section 2, we provide some background on the syntax-semantics framework that we employ and relate it to previous approaches to $\mathrm{p}$-verbs in the tradition of lexical decomposition grammar. We illustrate our syntax-semantics interface with the discussion of the three examples of p-verbs in (2) in section 3. The focus of our analysis is on emphasizing the differences between the formal and conceptual constituents of the meaning of those p-verbs. Next, in section 4 , we relate the differences in the meaning of $\mathrm{p}$-verbs to the restrictions which these p-verbs impose on the selection of direct objects. We generalize the observations about divergence in selectional preferences with a statistical measure known as selectional preference strength in section 5 and discuss the results of a proof-of-concept corpus study in section 6 . We conclude in section 7 .

\section{Pervasive semantics}

\subsection{Decomposition in the lexicon}

The assumption that the representation of word meaning in the lexicon is structured (and not purely denotational) proved to be a fruitful starting point for the decomposition of meaning in the lexicon to conceptual structures such as 'semantic forms' (Bierwisch 2007, Wunderlich 2012), 'event structure templates' (Rappaport Hovav \& Levin 1998), 'dot-types' (Asher 2011, Pustejovsky 2001), 'frames' or 'scenarios' (Fillmore 1982, Hamm et al. 2006). But what all these approaches share is the assumption that word meaning is determined in the lexicon according to principles different from the principles which apply to the determination of sentence meaning in the syntax. It is the assumption of a principal difference between the structure of meaning in the lexicon and the structure of meaning in sentences which we think causes the gap between conceptual and formal meaning. In formal semantics, sentence meaning is determined by the compositional interpretation of the syntactic structure of the sentence. In lexical semantics, word meaning is determined by 'flat' conceptual structures built from a set of basic concepts or fundamental constituents of meaning. Denominal p-verbs in particular have been in the focus of interest for lexical decomposition approaches, where it is assumed that a noun is incorporated with a lexical process into an abstract verbal template (Kaufmann 1995, Stiebels 1998). As an illustration, consider the semantic form that (Stiebels 
1998, p. 289) proposes for the denominal spatial p-verb unterkellern (build a cellar under sth.) in (3), see also (Roßdeutscher 2011,2013a) for a comparison of lexical decomposition with the present approach.

(3) Lexical entry for unterkellern:

$\lambda y . \lambda x \lambda s . C A U S E(x, B E C O M E(P O S S(y, C E L L A R)))(s)$

$\wedge B E C O M E(L O C(C E L L A R U N D E R[y]))(s)$

The semantic form (3) involves six different conceptual predicates CAUSE, BECOME, POSS, CELLAR, LOC and UNDER. unterkellern itself does not indicate the arrangement of these predicates. Also, the meaning of the conceptual predicates must be given in terms of a pre-theoretic language grounded in assumptions about the structure of human cognition such that paraphrases of the meaning of unterkellern as provide an object $x$ with a cellar such that the cellar is located under $x$ can be provided a reasonable interpretation. It is also assumed that each of the conceptual predicates encodes a number of additional constraints on the type of arguments it takes, e.g. that for a cellar to be located under an object, this object must provide a region in its underground (see (4a)). Similarly, the combinatorics of conceptual predicates must prevent an incoherent combination as in (4b). Furthermore, the conceptual predicates must license only appropriate modifications and rule out examples such as (4c). Taken together, the constituents (conceptual predicates) and principles of meaning formation (cognitively motivated processes) in the lexicon are fundamentally different from those constituents (words) and principles of meaning formation (compositional interpretation of syntactic structure) that have been employed with great success in the analysis of sentence meaning.

(4) a. *ein Flugzeug unterkellern

an airplane under.prfx.cellar

b. *ein Haus überkellern

a house over.prfx.cellar

c. *ein Haus mit Wasser unterkellern

a house with water under.prfx.cellar

Acknowledging these differences in scope and motivation and grossly generalizing, formal semantics is concerned with how meaning is derived compositionally from sequences of words but not what the fundamental constituents of meaning are 
and how they pattern in words. Lexical semantics is concerned with how the fundamental constituents of meaning pattern in words under the assumption that the meaning of words must be explained with the help of non-linguistic conceptual knowledge. In the following, we propose that bridging the gap between formal and conceptual semantics can be accomplished in an account of word-formation in which there is no generative lexicon but word-formation is entirely syntactic and consequently, the same semantic principles apply to words and sentences.

\subsection{Pervasive Syntax}

In pervasive syntax approaches to word formation (e.g. Hale \& Keyser 1993, Marantz 1997, Alexiadou 2001, Borer 2005), the same syntactic principles are assumed to be at work below and above the 'word level'. Words are formed from 'roots', atomic, non-decomposable and category-neutral elements associated with encyclopedic knowledge. Roots combine with features to build larger linguistic elements. Consequently, the term 'lexical item' has no significance in the theory and nothing can be said to 'happen in the lexicon'. We take the idea of pervasive syntactic structure all the way down as an inspiration for the development of a similarly pervasive semantics. We assume a fairly standard minimalist syntax of phrase structure with move and merge (Chomsky 1995, Adger 2003) and that incorporation is governed by the head movement constraint (Travis 1984). We also assume a minimalist approach to argument structure, where argument structure is determined in the syntax (Hale \& Keyser 1993) and a structural parallelism across the nominal (cf. Alexiadou 2001), verbal (cf. Harley 2011) and prepositional (cf. Svenonius 2003) domain.

The basic - and fairly standard - syntax of denominal verbs which we take as the starting point for our discussion is given in (5), (6) and (7).

(5) eine Terrasse bedachen to roof a terrace

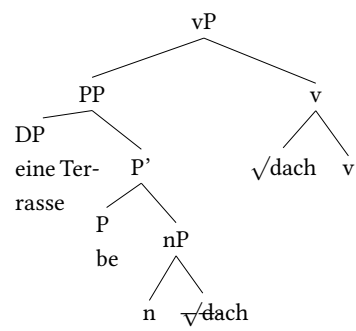

(6) eine Flasche lagern to store a bottle

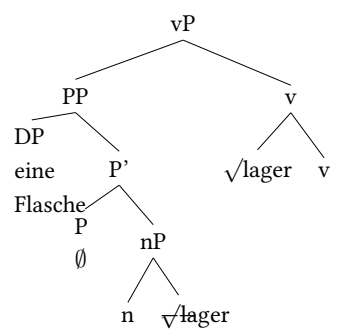

(7) einen Patienten stützen to support a patient

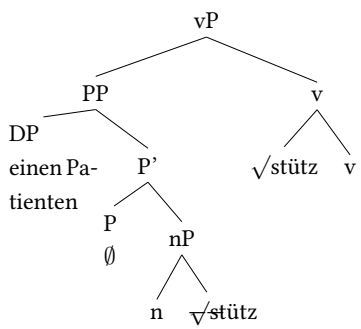


The structure of each of the examples (5), (6) and (7) evolves from the insertion of a root $\sqrt{ }$ into a nominal phrase template. The nominal phrase is merged with a prepositional head $\mathrm{P}$ which projects a phrase structure the specifier of which is a DP. Independent of whether $\mathrm{P}$ is overtly realized with the prefix $b e$ - (as in (5)) or not (as in (6), (7)) $\mathrm{P}$ has the same syntactic and semantic function. Finally, the prepositional phrase is merged with a verbalizer head $\mathrm{v}$, into which the nominal root incorporates via head movement.

\subsection{Pervasive Semantics}

In our approach of pervasive semantics, the semantics of (morphologically complex) words is not reconstructed in the lexicon but in the syntax. The starting point of our reconstructions is the insertion of a root into a syntactic context which determines the category of the root. The semantics of the root in that particular insertion context is incrementally specified by the semantic interpretation of the syntactic structure of the insertion context. That is, one and the same root can have different meanings, depending on the syntactic context in which it is inserted and interpreted. For example, the same root $\sqrt{ }$ lager can show up in the verb lagern (to store) and the noun Lager (the store), depending on the syntactic context into which $\sqrt{ }$ lager is inserted. As we have seen in the examples (5)-(7), syntactic contexts for root insertion have a functional structure determined by the layering of functional heads and their projections. In fact, functional heads have a categorizing function in the syntax we pursue. Heads of verbal phrases vP categorize verbs, heads of nominal phrases $\mathrm{nP}$ categorize nouns and heads of prepositional phrases PP categorize prepositions. The layering of functional structure also implies that in "a 'pervasive syntax' approach to morphologically complex forms $[\ldots]$ the analysis and structures proposed for a form must also be contained within the analysis of any structure derived from that form" (Harley 2009, p.320).

The hierarchy and modular organization of functional structure determined in the syntax requires a similar organization of the compositional semantic interpretation of the syntactic structure. Consequently, we propose that each functional head in the syntax is responsible for the introduction and predication of a particular sort of discourse referents. Put another way: functional layers in the syntax correspond to the ontological building blocks of word meaning. For example, $\mathrm{v}$ introduces events: $e, \mathrm{P}$ introduces states: $s, \mathrm{n}$ introduces invididuals: $x$, 
Place introduces regions (sets of bounded directed vectors): $r$ and $\mathrm{K}$ (ase) introduces Eigenspace-vectors: $r_{i d}$ (Wunderlich 1991). We also propose that the same close-knit connection between syntax and semantics holds for the introduction of conceptual predicates such as that between an event and its result state, i.e. the conceptual predicate CAUSE. Roßdeutscher \& Kamp (2010) argue that the syntactic configuration which gives rise to the CAUSE predicate is one in which a verbalizer $\mathrm{v}$ is merged with a state-denoting XP to the effect that the event introduced by $e$ is conceptualized as that event of which the state denoted by the XP is its result. Of particular interest to this paper are those conceptual relations that arise from the syntactic configuration of a merge of a P head with an XP, among them the application of one object to another object APPLICATION, the support of one object by another object SUPPORT, and the relative location of an object AT. To identify the conditions for the introduction of conceptual predicates from a merger of $\mathrm{P}$ and an XP, we need to make precise what exactly it is that application, support or location is a conceptualization of, i.e. how the denotation of the XP with which $\mathrm{P}$ merges influences the conceptual predication over the merge of $\mathrm{P}$ and the XP. To this end, we propose to take into account that the denominal verbs which we focus on in this paper involve an additional meaning component. Verbs like überdachen or einlagern identify a spatial configuration of the nominal root of the verb and the direct object of the verb. For example, überdachen in (8a) describes an event in which an object - the roof - is brought into the region above some other object - the terrace. einlagern as in (8b) describes an event in which an object - the bottle is brought into a location inside of another object - the store. abstützen as in (8c) describes an event in which an object - the truss - is provided with pillars in its below region. 
(8) a. eine Terrasse überdachen

a terrace over.prfx.roof

to roof a terrace

b. eine Flasche einlagern

a bottle in.prtc.store

to store a bottle

c. einen Dachstuhl abstützen

a truss up.prtc.stilt

to prop up a truss
(9)

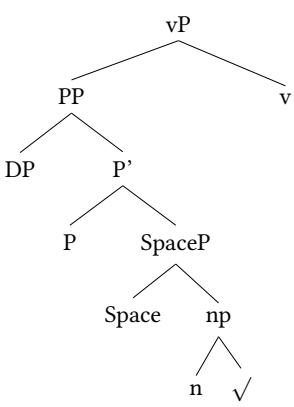

Spatial configurations of the type described in (8a)-(8c) can be represented as conditions on vector spaces: (Zwarts 1997, 2005, Zwarts \& Winter 2000) proposed a formal semantics for spatial expressions built from vector spaces in which the denotation of objects is their Eigenspace and spatial configurations are formally defined in terms of structural constraints on sets of vectors such as spatial inclusion (represented as " $\subseteq$ ") or being a set of vectors which point upwards from a reference object $x$ (represented as “ $\uparrow(x)$ ”), giving the 'above region' of $x$. For example, in terms of vector space semantics, (8a) is true iff the Eigenspace of the roof used to cover the terrace is located in the above region of the terrace and the above region of the terrace is covered by the Eigenspace of the roof. Similarly, (8b) is true iff the Eigenspace of the bottle is a subset of the vectors defining the interior space of the store. Finally, (8c) is true iff the Eigenspaces of the pillars have contact with and are located in the below region of the truss.

It is obvious even from these informal elaborations that just the spatial configurations described by (8a)-(8c) are not sufficient as characterizations of the meaning of (8a)-(8c). What is necessary in addition is a conceptualization of the spatial configuration as a configuration of support, application or inclusion. Earlier we said that the conceptualization of support, application or inclusion is realized with the merge of $\mathrm{P}$ and an $\mathrm{XP}$ and we are now in a position to make more precise what the XP with which $\mathrm{P}$ merges is about. $\mathrm{P}$ merges with an XP describing a spatial configuration. To keep the formal characterization of the spatial configuration in terms of vector space semantics apart from the conceputalization of a spatial configuration as a certain relation holding between objects, we call the functional head of the XP with which P merges 'Space'. The syntactic structure of denominal verbs taking into account their spatial semantics is thus a refinement of 
the basic structure in (5), (6) and (7): it contains an additional functional layer SpaceP inbetween the functional PP layer and the root $\mathrm{nP}$, see (9).

The syntactic structure in (9) provides two main switching points for the semantic interpretation. On the one hand, there is the Space functional layer responsible for the computation of the spatial configuration of vectors described by the verb. On the other, there is the $\mathrm{P}$ functional layer responsible for the conceptualization of the spatial configuration of vectors as a certain conceptual relation between objects. The difference is that not any vector space object can be conceptualized as a roof or a terrace, because a roof or a terrace is more than just their geometry and location, a roof is associated with a certain concept and so is a terrace. Conceptually, a roof is "a protective covering that covers or forms the top of a building" (Wordnet search, Fellbaum 1998) and a terrace is a "usually paved outdoor area adjoining a residence" (Wordnet search). That is, the function of $\mathrm{P}$ conceptualizing a spatial configuration is to check whether the concepts associated with the vector-space object can be coherently predicated as standing in a conceptual relation of support, application or inclusion based on the contribution of SpaceP. This is the syntactic 'locus' where the incoherent examples in (4a), (4b) and (4c) are filtered out. The structural split of formal and conceptual aspects of meaning has two welcome consequences. First, formal and conceptual aspects of meaning are not located in different places as in customary approaches that distinguish a lexicon and the syntax-semantics interface. Second, the unified treatment of formal and conceptual aspects of meaning in the same system of linguistic interpretation allows to assess the distinction between formal and conceptual aspects of meaning from a perspective that is based on linguistic evidence rather than on the distinction between lexicon and sentence that must be motivated by different evidence, e.g. assumptions about the architecture of the human cognitive system à la lexical decomposition grammar. Before we explore the issue of the relation between formal and conceptual semantics in full detail in section 4, we now turn to an in-depth analysis of three examples of spatial denominal p-verbs.

\section{Example analyses}

\section{1 überdachen}

The first example of a denominal spatial p-verb which we would like to discuss in more detail is überdachen as in (10). (10) is exemplary for a class of spatial denominal 
p-verbs involving a conceptual relation of application. This class includes verbs such as ummauern (to wall), überpflastern (to cobble), umzäunen (to fence in), aufstocken (to ramp up), überdeckeln (to cover with a lid), überdecken (to cover), untertunneln (to tunnel under) and überbrücken (to bridge).

(10) eine Terrasse überdachen

a terrace over.prfx.roof

The reconstruction (11) of (10) at the syntax-semantics interface contains only the main steps of interpretation and is thus grossly simplified. In particular, we use free variables in the lower parts of the structure that would enter the representation only higher up in a compositional analysis. The representations we use are to be understood in the spirit of those representations used in Discourse Representation Theory (Kamp et al. 2011). For überdachen and the next example einlagern, a detailed reconstruction making explicit all step of composition is given in the appendix. In (11), all constituents in the syntactic representation are in situ. Under the assumption of a functional split between formal and conceptual semantics in the syntax, we distinguish two aspects of the compositional semantic structure of p-verbs. 
(11) eine Terrasse überdachen

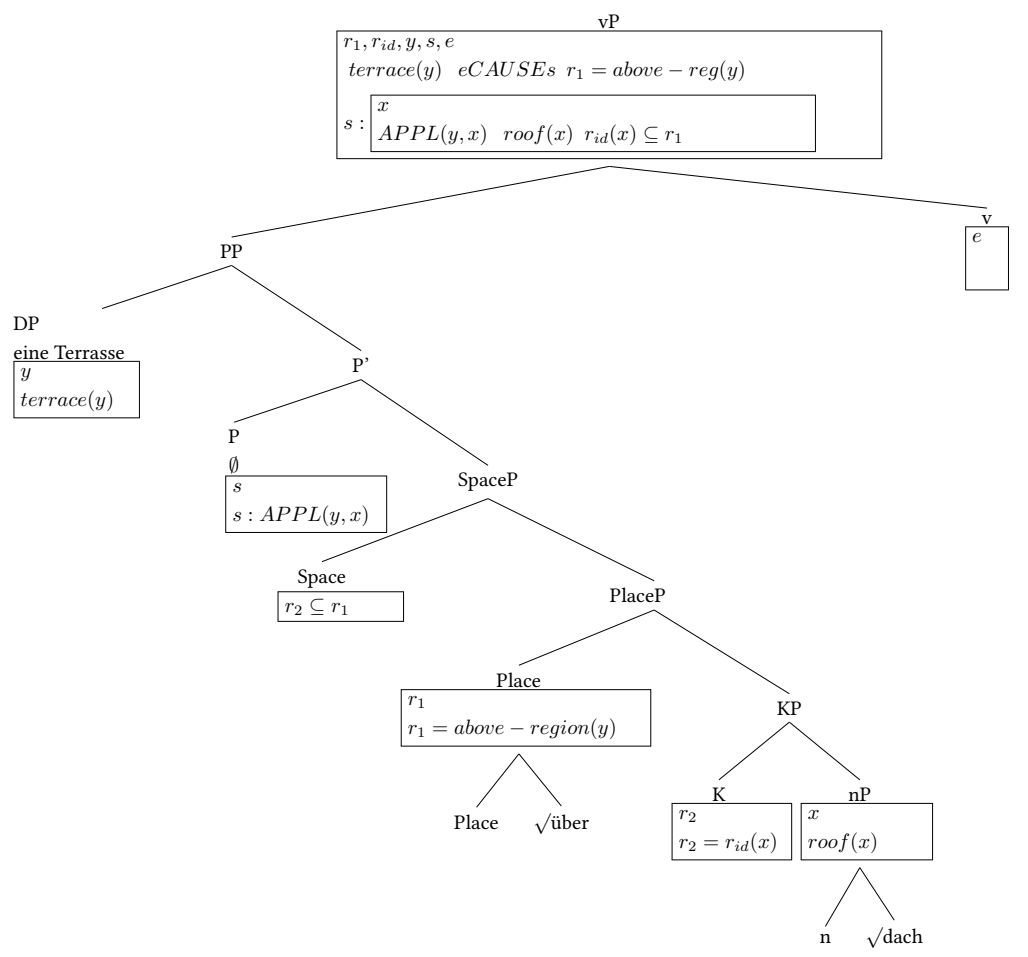

Starting at the bottom of the representation, the root $\sqrt{ }$ über introduces a region (indicated by the head Place) which is the above-region of the terrace. The root $\sqrt{ }$ dach is inserted into a $\mathrm{nP}$ context which is selected by KP so as to reconstruct the Eigenspace $r_{i d}$ of the entity denoted by $\mathrm{nP}$. SpaceP relates the region denoted by PlaceP with the Eigenspace denoted by $\sqrt{ }$ dach + KP so as to express the spatial truth-conditions expressed by the phrase consisting of the p-verb and its direct object, i.e. that the Eigenspace of $\sqrt{ }$ dach is contained in the above region of the reference object.

On top of SpaceP, P is responsible for conceptualizing the vector space semantics calculated at SpaceP. In the present example, $\mathrm{P}$ conceptualizes the containment of the Eigenspace of $\sqrt{ }$ dach in the above region of the reference object as the application of a roof to the reference object. Conceptualization of the abstract truth-conditions at SpaceP as an instance of application requires that roofs and terraces are not just geometrical objects. In order to enter the conceptual application 
relation in a coherent way, the geometrical objects representing terrace and roof must be conceptualized as a terrace or roof. We will discuss our implementation of conceptual coherence in more detail in section 4 .

Finally, the representation of the vP-node is to be read as follows: the statedenoting $\mathrm{PP}$ is merged with $\mathrm{v}$, giving rise to the conceptualization as $e C A U S E s$, i.e. that the result state of the event of application described consists in the terrace $\mathrm{y}$ having a roof $\mathrm{x}$ and that the eigenregion of the roof, $\mathrm{r}_{i d}(\mathrm{x})$, is a included in the above-region $\mathrm{r}_{1}$ of the terrace. The variable $\mathrm{x}$ and its characterising condition roof(x) are part of an inaccessible sub-DRS, representing incorporation.

What is important for the argument we want to put forward in this paper is that both conceptual and formal aspects of meaning are encoded by the same principles of semantic composition. Consequently, the difference between conceptual and formal meaning does not manifest in the same way as it does in the opposition between lexical and sentence meaning. Rather, what the analysis of überdachen suggests is that the distinction of conceptual and formal meaning is more finegrained than the binary lexical vs. sentence distinction. In particular, the way in which we represented the semantics of überdachen encodes both formal and conceptual aspects of meaning linguistically, i.e. without recurse to a languageindependent structuring of conceptual predicates. For überdachen, we located the contribution of conceptual meaning in the constraints that conceptualization puts on the interpretation of formal meaning, i.e. in the selection of appropriate denominal roots, prefixes and direct objects. überdachen constitutes a case in which such selection restrictions are relevant to all constituents of verbal phrases in which überdachen occurs. We will see in the next two examples that this does not always need to be the case.

\section{2 einlagern}

The next example which we would like to discuss in more detail is einlagern (to store). (12) is exemplary for a class of p-verbs involving the conceptual relation of location, among them einsacken (to bag sth.), einsperren (to cage), einkellern (to store), einkerkern (to incarcerate).

(12) eine Flasche (in den Keller) einlagern

a bottle (in the cellar) in.prtc.store

put a bottle in the cellar 
einlagern is a particle verb, see (13).

(13) Peter lagert die Flasche ein

Peter store.V a bottle in.prtc

Peter stores a bottle

Syntactically, the particle verb einlagern has a particle-phrase $p \mathrm{P}$ on top of the denominal vP, see (15). This construction prevents the incorporation of the particle ein into the verb via the head movement constraint. The $p \mathrm{P}$ contributes the information that the bottle becomes stored inside a location. It should be noted that even if the location in which the direct object of einlagern ends up is not mentioned explicitly, it is nevertheless presupposed part of the meaning of einlagern that there is a distinct location inside of which the object to be stored ends up. The interior space of the denominal root can be picked up with a locative PP such as in den Keller (in the cellar), compare (12). To see why the constraint that the final location of the direct object ends up inside the space provided by the nominal root is contributed by the particle ein, consider the verb lagern without the particle as in (14).

(14) Peter lagert Holz auf dem Boden

Peter store.V wood on the ground

Peter stores wood on the ground

Lagern on its own does not come with the requirement that its direct object must be located inside the space provided by its nominal root, because any distinct place will be suitable to store an object. Geometrically, lagern requires that its direct object is located in space relative to another object or landmark. This boils down to the requirement that the direct object has an Eigenspace and that this Eigenspace can be located in space. In contrast, the contribution of ein in einlagern is that it additionally requires that the direct object is located inside a store and not just at a certain location. That is, the difference between lagern and einlagern is that lagern only requires a specified location of the direct object where it remains for some contextually specified time whereas einlagern makes explicit that the direct object is moved into a certain place. Consequently, in the $p \mathrm{P}$ structure, we have a figure-ground relation between the bottle and the cellar, where the bottle ends up in the cellar. 
Semantically, the specific syntax of the particle construction in which the contribution of the particle ein is considered only above the denominal vP leads to a configuration in which there are two states $s_{1}$ and $s_{2}$ responsible for the conceptualization of two dependent geometrical configurations. $s_{2}$ represents that state which conceptualizes the location of the direct object with respect to the denominal root. $s_{1}$ further specifies this location as a location inside the space provided by the denominal root. However, the states $s_{1}$ and $s_{2}$ are result states of the same event event $e$. That is, particle constructions of the type exemplified by einlagern involve a 'double predication' of the result state relative to the denominal root. We thus assume that $s_{1}$ and $s_{2}$ are unified as results of the event defined by the merge of $p \mathrm{P}$ and $\mathrm{vP}$ but are semantically distinct.

What is important to the goals of this paper is that in (12) the conceptualization of the geometry with $L O C$ resp. $I N$ does not impose restrictions which are not already structurally conveyed at SpaceP, namely that the location of the bottle is fixed with respect to a certain region or place: $a t\left(r_{1}, r_{2}\right)$ and that the Eigenspace of the bottle is included in the Eigenspace of the store $r_{1} \subseteq r_{2}$. In other words, unlike in (überdachen), the conceptual meaning of einlagern does not effect interpretation in the form selection restrictions on possible fillers of the argument slot for the direct object. 
(15) eine Flasche in einen Keller einlagern

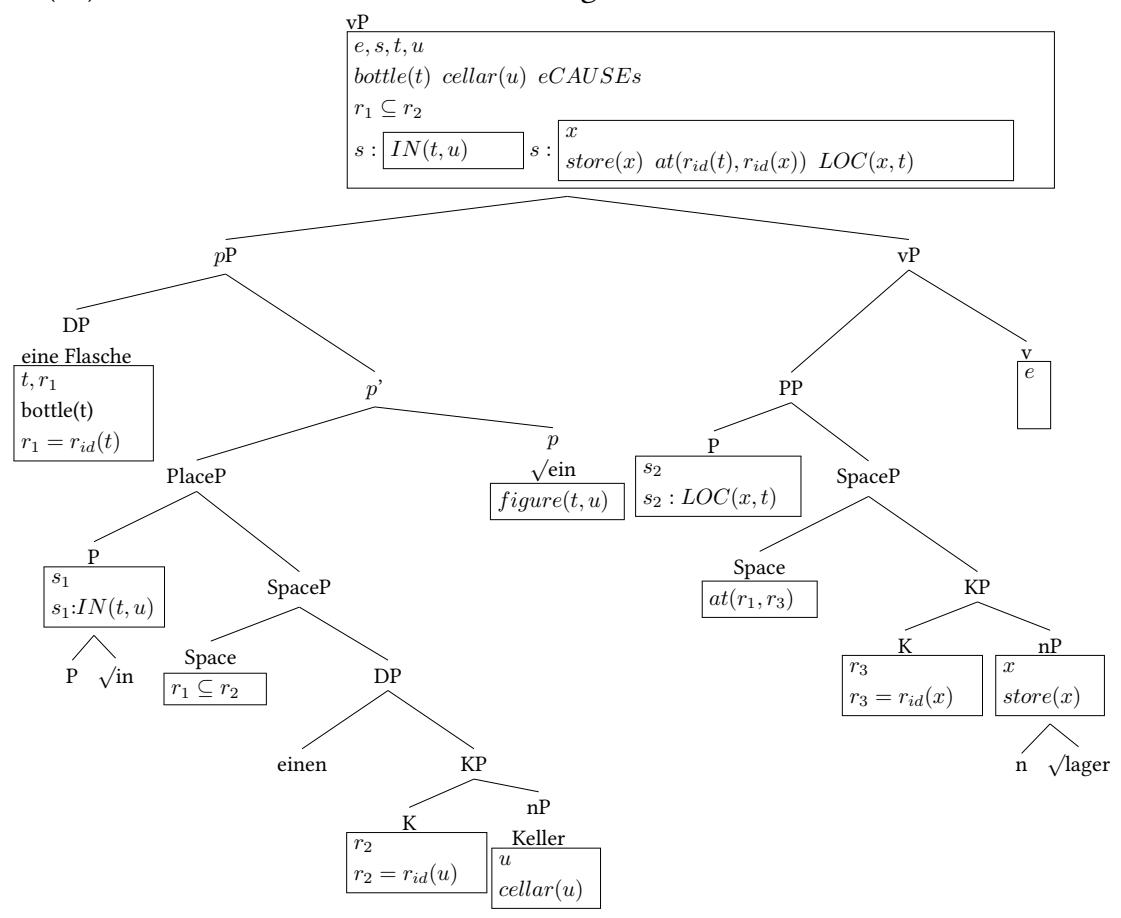

\section{3 abstützen}

The final example (16) is exemplary of a class of denominal p-verbs which involves the conceptual relation of SUPPORT, among them aufbocken (to jack up), verstreben (to strut), untermauern (to support), unterfüttern (to reline), aufkanten (to tilt sth.) and aufbahren (to lay sb. out).

(16) einen Dachstuhl abstützen
a truss under.prtc.stilt
to prop up a truss

Like einlagern, abstützen is a particle verb and thus has a similar syntax and semantics in which a particle phrase is merged with a denominal verb phrase and the denominal root is subject to double predication by both the verb and the particle. Despite these structural similarities, the the contribution of the particle 
structure with $\sqrt{ }$ ab as its prepositional element is of a different nature than the contribution of ein in einlagern.

We propose that the geometrical relation involved in the reconstruction of (16) is a relation of contact between an object $x$ and a face $r$ of another object. We represent contact between $x$ and $r$ as $\left.x @ r \rightsquigarrow\left(r_{i d}(x) \bigcap r \neq \emptyset\right)\right)$. But for the conceptualization of SUPPORT, geometrical contact between objects is not enough because there are lots of geometrical contact relations which are not relations of support, e.g. a bubblegum adhering at the bottom of a table has contact with a face of the table but it does not support the table. Instead, the conceptualization of SpaceP with the relation $S U P P O R T$ between the nominal root $\sqrt{ }$ stütz (pillar, stilt), the particle $a b$ and the direct object Dachstuhl is quite complex in (17).

(17) einen Dachstuhl abstützen

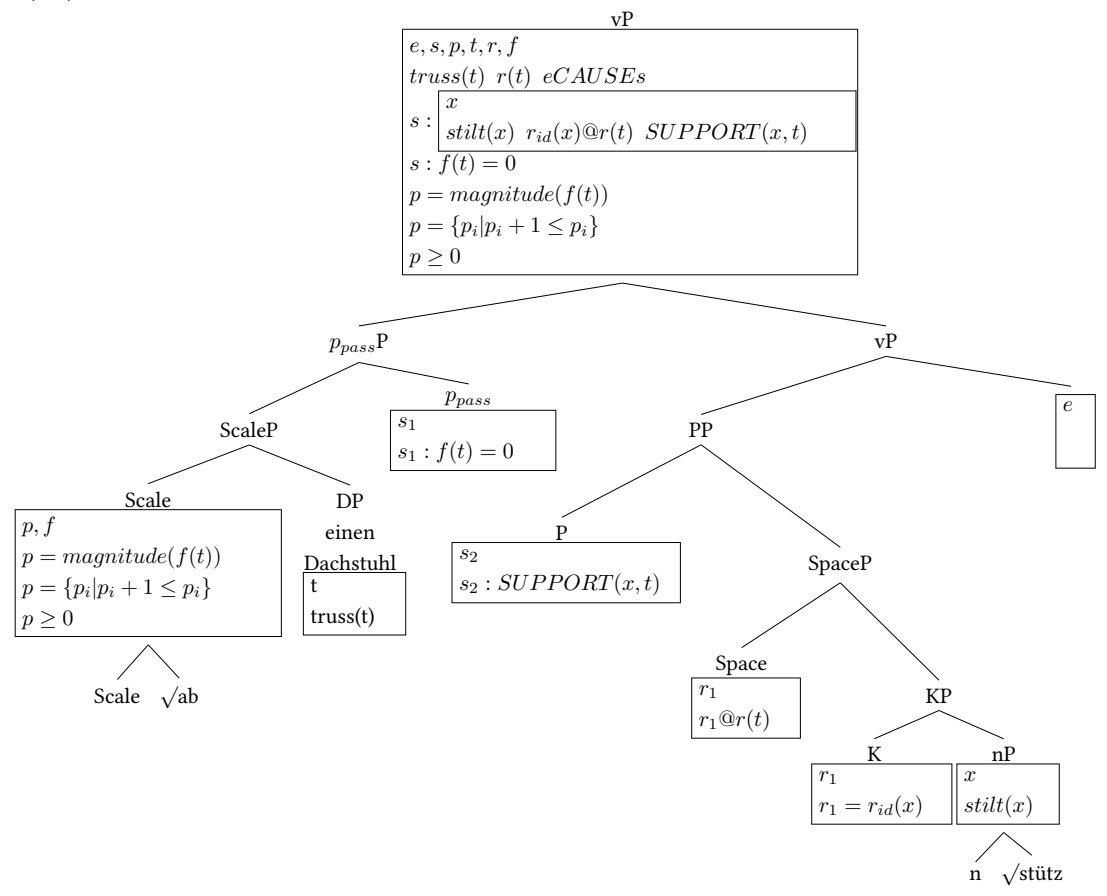

To identify the specific contribution of the particle $a b$, let us consider the $\mathrm{vP}$ branch of the structure representing the contribution of the nominal root $\sqrt{ }$ stütz. The vP branch derives the verb stützen (to support) as in (18). 
(18) einen Patienten stützen

a patient stilt.V

to support a patient

stützen does not imply that the force exerted on the direct object is completely absorbed but the direct object itself absorbs some portion of the forces exerted on it. In contrast, the contribution of the particle $a b$ in abstützen is that the forces exerted on the direct object are completely absorbed in the course of the event described. Conceptually, we model this contribution of $a b$ in terms of $a b$ contributing a decreasing scale $p=\left\{p_{i} \mid p_{i}+1 \leq p_{i}\right\}$ of magnitudes of the net force $f(t)$ exerted on the direct object $t$. From this point of view, what abstützen describes is an event which is made up from a sequence of change of states each of which results in a lower point on the scale of net forces exerted on the truss. In plain words, abstützen describes an event of incremental reduction of the net force exerted on its direct object. This event is bounded by a particular state, namely that state in which the net force exerted on the truss becomes zero. The approach we just sketched receives further support from constructions in which the incremental nature of the supporting event and its boundedness is made explicit with a mit/(with)-PPs involving genügend (sufficient) as a description of the event boundary, see (19)

(19) den Dachstuhl mit genügend Balken abstützen

the truss with sufficient timber under.prtc.stilt

to support a truss sufficiently with timber

We render the intuitions about the contribution of the $a b$-particle with a construction in which a $p_{\text {pass }}$ head quantifies over a sequence of states of decreased net force and the event modified with $a b$ pertains to the sum of the states quantified by $p_{\text {pass }}$. Formally, our analysis in (17) is based on (Roßdeutscher 2012,2013b)'s proposal for such 'passive' $p$ constructions. In analogy to verbal passives where the agent is demoted and the theme is promoted, in passive $p$-constructions the figure is demoted and the ground is promoted. For einlagern, the direct object is the figure whereas the nominal root plays the role of the ground. In abstützen, there is no explicit figure. Instead, the ground (if we would still call it like this) - the truss - is promoted as the direct object of abstützen: different from einen Keller in (15), which receives prepositional accusative case, einen Dachstuhl does not and leaves the $p_{\text {pass }}$-phrase in order to receive accusative case in vP. 
The semantic effect of $p_{\text {pass }}$ is a quantification over the elements of the implicit resp. demoted figure, i.e. the decrease in net force on the truss that the timber stilts that are moved into the below-region of the truss bring with them. The effect of this quantification is boundedness of the event description: the totality of timber stilts exerts a force on the truss which renders the net force on the truss zero and thus, as a result of the event described, the truss is supported in upholding against gravity. (17) represents the semantic constribution of $p_{\text {pass }}$ in a simplified manner, leaving out the details of the quantification over states represented on the scale of net forces exerted on the truss. Additional complexity is introduced in the analysis by the fact that abstützen is, just like einlagern, a particle verb and thus the semantics involves the same kind of 'double-predication' of the result state of the event predicated in vP. That is, similar to the predication of the nominal root $\sqrt{ }$ lager in einlagern (12), the mit-PP in (19) reintroduces the incorporated nominal root $\sqrt{ }$ stütz.

What is important to note from the discussion of abstützen and the involved conceptualization with SUPPORT is that formal meaning is by far not enough to capture what the conceptual meaning of SUPPORT is about. For SUPPORT, the additional conceptual machinery of force dynamics has to be invoked to grasp the meaning of constructions involving abstützen, which sets it apart from the relation between formal and conceptual meaning in both einlagern and überdachen, an observation which we explore in full detail in the next section.

\section{Selection Restrictions}

With respect to the case under consideration, in our discussion of the relation between formal and conceptual semantics, we focus on conceptual coherence pertaining to the restrictions imposed on the selection of appropriate fillers of a conceptual relation, in the case under discussion the nominal root of the verb and the direct object of the verb. For example, not any objects will afford the selection restrictions involved in überdachen imposed by the application relation. Basically, there are two cases to be distinguished. First, it may be the case that the nominal root of the verb fails to satisfy the selection restrictions imposed by the conceptual application relation as in (20). 
(20) ?eine Terasse unterdachen

a terrace under.prfx.roof

While unterdachen as in (20) is superficially similar to überdachen, there is an important conceptual difference between the two. If a roof is conceptualized as being an object in the above region of the object which it protects, then the combination of dach with $\ddot{u} b e r$ is conceptually coherent. But for (20), this conceptualization runs into a problem: a roof cannot be conceptualized as being in the below region of the object with respect to which it is conceptualized as a roof. That is, although unterdachen is perfectly acceptable from a formal point of view, conceptualization rules out unterdachen as a possible word. The second case of selection restrictions applies to the conceptualization of überdachen with respect to the direct object Terasse. This conceptualization requires that the direct object can be conceptualized as an object which provides a bounded 'above-region' in order for the roof to be applied: a terrace fulfills these restrictions whereas a basement does not. einen Keller überdachen as in (21) is conceptually incoherent because a basement is usually not conceptualized as providing an above region in which another object can be placed, and thus selection restrictions rule out Keller as a suitable direct object of überdachen.

\section{(21) *einen Keller überdachen}

\section{a basement over.prfx.roof}

The argument that we develop in the following pertains to the relation between the degree of selectivity on direct objects imposed by the conceptual relation involved in the reconstruction of a p-verb and characterization of the three different types of conceptual relations that we introduced with our examples: (a) a conceptual relation of support as in abstützen, (b) a conceptual relation of application as in überdachen and (c) a conceptual relation of location as in einlagern. When we reconsider the relation between the geometric truth-conditions, i.e. the spatial configurations expressed by each of these verbs and the conceptual relation involved, then it appears that each of the verbs exemplifies a different proportion between the role of conceptual and formal meaning. For the geometrical relation of location inside a region as in einlagern, conceptualization with LOC does not impose any additional constraints on direct objects which are not already conveyed in terms of geometrical constraints. What is important to einlagern is only that 
the direct object fits into the space provided by the store, not conceptual properties of the direct object. For überdachen, conceptual properties of the direct object are relevant: as we saw with Keller, the direct object must be associated with a concept that provides a bounded above region into which the roof can be applied. Consequently, selection restrictions play a role for überdachen in that only a certain class of objects will be accepted. Finally, the strongest conceptual contribution can be found with support relations as in abstützen. The conceptualization of support involves conceptual properties that allow for the computation of forces and as such involve additional knowledge about gravitation and physics. Consequently, support p-verbs are quite restrictive with respect to their possible direct objects: e.g. direct objects must not absorb gravitational forces on their own in order to be propped up. These observations on the divergence between geometry and concepts in p-verbs suggest a measure on the relation between formal and conceptual semantics as follows.

Any well-formed logical form has an interpretation but not any interpretation of a well-formed logical form is conceptually coherent. Logical forms (whatever their extension is, individuals or geometrical objects) employed in truth-conditional semantics are insensitive to conceptual coherence. What distinguishes formal and conceptual semantics in our approach is not the distinction between lexicon and sentence but their respective contribution to the meaning of a construction. That is, if selection restrictions (i.e. restrictions pertaining to content) are the contribution of conceptual semantics and truth-conditions (i.e. restrictions pertaining to structure) are the contributions of formal semantics, then the relation between formal and conceptual semantics shows up in the contribution of selection restrictions on the fillers of argument positions of a logical form: selection restrictions reflect the contribution of conceptual semantics in the instantiation of a logical form. Consequently, the stronger conceptual restrictions are imposed on the selection of fillers of argument slots of logical forms, the more emphasis is put on conceptual structures in the meaning of the logical form. This hypothesis has a direct reflection in our pervasive approach of semantics. Instead of a divide between conceptual meaning in the lexicon and truth-conditional meaning in sentences, in our approach there is a continuum of relations between truth-conditions and conceptual structure with verbs such as einlagern focusing formal semantics and structural constraints on the one and verbs such as abstützen focusing conceptual semantics and selection restrictions on the other end. 
Given the argument of the last paragraph, we expect that if application, support and inclusion are different conceptual relations, this difference shows up in terms of different selectional preference strength. That is, we expect that there is a correlation between the conceptual relation involved in the reconstruction of word meaning and the selectional preference strength of the verb. Consequently, we can measure the relation between formal and conceptual semantics in considering the selectional strength of conceptual relations against the insensitivity of logical forms to conceptual coherence. In our examples, we predict that conceptual relations are ordered according to their selectional preference strength, from strong to weak: $S U P P O R T>A P P L>L O C$. We saw that $L O C$ in einlagern does not involve conceptual restrictions which are not already captured by the truth-conditions of geometrical inclusion: for putting an object in a store, it does not matter which concept is associated with the object to be stored as long as the geometry of the stored object can be included in the geometry of the store. We also saw that $A P P L$ in überdachen involves a relevant conceptual restriction on the objects standing in the application relation which is not captured by the truth-conditions of geometrical inclusion: the direct object must have an above region with distinct boundaries. Finally, SUPPORT in abstützen does not only involve conceptual constraints on the objects which stand in the support relation but also requires to take into account the additional concept of force dynamics. abstützen requires appropriate direct objects to be possible subject to the laws of gravity and to provide a below region.

\section{A statistical measure for selectivity}

The point we want to make with our analysis is the following: in our framework, there is a linguistic measure for the relation between formal and conceptual semantics in terms of selection restrictions, which exemplify the relation between conceptual semantics sensitive to conceptual coherence and truth-conditions insensitive to conceptual coherence. According to our proposal, if conceptual relations manifest linguistically in the strength of selection restrictions and selectional association, conceptual predicates may be considered as a stepping stone towards the linguistic exploration of conceptual meaning. In our approach, conceptual meaning can be defined linguistically without reference to conceptual structures in the first instance. Instead, our notion of conceptual meaning paves the way 
to a classification of concepts based on empirical observations (for p-verbs see e.g. Rüd 2012, Springorum et al. 2012), where conceptual predicates are labels for degrees of selection preference strength. Empirically, our hypothesis can be tested with the help of the observation of the relative entropy of verbs and the conceptual class of their direct objects as proposed by Resnik (1996). Resnik (1996) approaches selection restrictions as the degree to which a pair of a verb and a syntactic relationship, here direct object, constraints possible conceptual classes of fillers of the argument slots of the syntactic relationship.

The intuition behind Resnik's selectional preference strength (SPS) is that a verb-relation pair that only allows for a limited range of direct objects will have a posterior distribution of conceptual classes of direct objects in which the verb is taken into account that strongly diverges from the prior distribution of conceptual classes of direct objects in which the verb is not taken into account. In order to quantify the degree of restrictions in a verb-relation pair, the overall probability distribution of noun classes is compared to the distribution of noun classes in the direct object position of the verb. Technically, this is achieved by calculating the relative entropy (the Leibler-Kullback divergence) $D$ of two distributions, the prior distribution $P(c \mid r)$ and the posterior distribution $P(c \mid v, r)$. The parameters $P(c \mid r)$ and $P(c \mid v, r)$ can be estimated from the corpus frequencies of tuples $(v, r, a)$ and the membership of nouns $a$ in GermaNet classes $c$.

$$
\begin{aligned}
S P S(v, r) & =D(P(c \mid v, r) \| P(c \mid r))) \\
& =\sum_{c \in C} P(c \mid v, r) \log \frac{P(c \mid v, r)}{P(c \mid r)}
\end{aligned}
$$

Resnik's approach relies on Wordnet for the generalization from direct objects to conceptual classes, but it should be noted that selection restrictions can be induced without lexical resources by using e.g. co-occurence for the generalization step (Erk \& Padó 2010). 


\section{Testing our predictions on Corpus Data}

$\begin{array}{llll}\text { Verb } & \text { Concept } & \text { SPS(4) } & \text { SPS(7) } \\ \text { einlagern } & \text { IN } & 0.1 & 0.2 \\ \text { einsperren } & \text { IN } & 0.7 & 0.9 \\ \text { überbrücken } & \text { APPL } & 0.6 & 2.0 \\ \text { überdachen } & \text { APPL } & 0.8 & 4.1 \\ \text { abstützen } & \text { SUPP } & 1.3 & 8.0 \\ \text { aufbocken } & \text { SUPP } & 1.7 & 4.9\end{array}$

Table 1: Selectional Preference Strength SPS(n) for selected p-verbs with respect to mappings of direct objects to GermaNet Classes of level $n$, counting levels from the top-level concept.

To give the reader a first impression of how Resnik's Selectional Preference Strength relates to our predictions, we conducted a proof-of-concept study. First, we estimated the prior distribution of nouns occuring in the direct object position of verbs mapped to GermaNet Classes (Hamp \& Feldweg 1997) from the first 200.000.000 sentences of SdeWac (Faaß \& Eckart 2013). Second, we extracted pairs of p-verbs and their direct objects with accusative case from SdeWac, manually disambiguated the set of direct objects to those objects which do not imply a metaphorical or non-spatial usage of the verb and mapped the remaining direct objects to GermaNet Classes in order to calculate the posterior probability of a GermaNet Class to occur in the direct object position of a p-verb. Table 1 shows the results for some of the verbs for which we were able to acquire enough instances which were covered by GermaNet. The higher the SPS of a verb, the more restrictions it imposes on possible fillers of its direct object argument slot. Intuitively, the data in table 1 reproduces our predictions quite well. P-verbs such as aufbahren or aufbocken are quite restrictive with respect to the type of direct objects they accept. In fact, aufbocken selects for land vehicles and abstützen selects for physical objects such as buildings. einlagern and einsperren on the other hand select for a wide range of GermaNet classes of direct objects and thus receive a lower SPS number. 


\section{Summary}

We introduced a pervasive approach to semantics which does not postulate a structural distinction between lexicon and sentence. We proposed that in our framework, the relation between formal and conceptual semantics can be measured out empirically in terms of selectional preference strength.

\section{References}

Adger, D. 2003. Core syntax. Oxford: Oxford University Press.

Alexiadou, A. 2001. Functional structure in nominals. Nominalization and ergativity. John Benjamins.

Asher, N. 2011. Lexical meaning in context: A web of words. Cambridge: Cambridge University Press.

Bierwisch, M. 2007. Semantic form as interface. In A. Späth (ed.). Interfaces and interface conditions. 1-32. de Gruyter.

Borer, H. 2005. Structuring sense. Vol. I \& II. Oxford: Oxford University Press.

Chomsky, N. 1995. The minimalist program. Cambridge, MA: MIT Press.

Cooper, R. 1983. Quantification and syntactic theory. Dordrecht: Reidel.

Erk, K. \& S. Padó. 2010. A flexible, corpus-driven model of regular and inverse selectional preferences. Computational Linguistic 36(4). 723-763.

Faaß, G. \& K. Eckart. 2013. SdeWaC - a corpus of parsable sentences from the web. In I. Gurevych, C. Biemann \& T. Zesch (eds.). Language Processing and Knowledge in the Web. Proceedings of the 25th International Conference, GSCL 2013. LNAI. Springer.

Fellbaum, C. 1998. Wordnet: An electronic lexical database. Bradford Books.

Fillmore, C. J. 1982. Frame semantics. In T. L. S of Korea (ed.). Linguistics in the morning calm. Selected papers from SICOL-1981. Seoul: Hanshin.

Hale, K. \& S. J. Keyser. 1993. On argument structure and the lexical expression of syntactic relations. In K. Hale \& S. J. Keyser (eds.). The view from building 20: Essays in linguistics in honor of Sylvain Bromberger. Cambridge, MA: MIT Press.

Hamm, F., H. Kamp \& M. van Lambalgen. 2006. There is no opposition between formal and cognitive semantics. 
Hamp, B. \& H. Feldweg. 1997. GermaNet - a lexical-semantic net for German. In Proceedings of the ACL workshop automatic information extraction and building of lexical semantic resources for NLP applications. Madrid.

Harley, H. 2009. The morphology of nominalizations and the syntax of vP. In A. Giannakidou \& Rathert M. (eds.). Quantification, definiteness, and nominalization. Oxford University Press.

Harley, H. 2011. A minimalist approach of argument structure. In C. Boeckx (ed.). The Oxford handbook of linguistic minimalism. Oxford University Press.

Kamp, H., J. van Genabith \& U. Reyle. 2011. Discourse Representation Theory. In D. M. Gabbay \& F. Guenthner (eds.). Handbook of philosophical logic. Vol. 15. 125 - 394. Springer $2^{\text {nd }}$ edition.

Kaufmann, I. 1995. Konzeptuelle Grundlagen semantischer Dekompositionsstrukturen. Die Kombinatorik lokaler Verben und prädikativer Komplemente. Vol. 335. Tübingen: Niemeyer.

Kratzer, A. 1996. Severing the external argument from its verb. In J. Rooryck \& L. A. Zaring (eds.). Phrase structure and the lexicon. 109-137. Dordrecht: Kluwer Academic Publishers.

Marantz, A. 1997. No escape from syntax: Don't try morphological analysis in the privacy of your own lexicon. U. Penn Working Papers in Linguistics 4.2.

Pustejovsky, J. 2001. Type construction and the logic of concepts. In F. Busa (ed.). The language of word meaning. 91-123. Cambridge: Cambridge University Press. http://dx.doi.org/10.1017/CBO9780511896316.009.

Rappaport Hovav, M. \& B. Levin. 1998. Building verb meanings. In M. Butt \& W. Geuder (eds.). The projection of arguments: Lexical and compositional factors. 97-134. Stanford: CSLI.

Resnik, P. 1996. Selectional constraints: an information-theoretic model and its computational realization. Cognition 61. 127-159.

Roßdeutscher, A. 2011. Particle verbs and prefix verbs in German: Linking theory versus word-syntax. Leuvense Bijdragen 97.

Roßdeutscher, A. 2012. Hidden quantification in prefix and particle verbs. In Proceedings of Sinn und Bedeutung 16. Vol. 2. 513-526. Utrecht: MIT WPL.

Roßdeutscher, A. 2013a. Denominal spatial prefix-verbs revisited. In A. Roßdeutscher (ed.). Sub-Lexical Investigations: German particles, prefixes and prepositions. Working Papers of the SFB 732 (SinSpec). Vol. 11. 58-88. University of Stuttgart. 
Roßdeutscher, A. 2013b. A syntax-semantics interface for P-elements in german verbal constructions. In A. Roßdeutscher (ed.). Sub-Lexical Investigations: German particles, prefixes and prepositions. Working Papers of the SFB 732 (SinSpec). Vol. 11. 1-57. University of Stuttgart.

Roßdeutscher, A. \& H. Kamp. 2010. Syntactic and semantic constraints in the formation and interpretation of ung-nouns. In Nominalisations across languages and frameworks. Berlin: Mouton de Gruyter.

Rüd, S. 2012. Untersuchung der distributionellen Eigenschaften der Lesarten der Partikel 'auf' mittels Clustering-Methoden. Unpublished master's Thesis. IMS, University of Stuttgart.

Springorum, S., S. Schulte im Walde \& A. Roßdeutscher. 2012. Automatic classification of German an particle verbs. In Proceedings of LREC-2012. Istanbul.

Stiebels, B. 1998. Complex denominal verbs in German and the morphologysemantics interface. Yearbook of Morphology. 265-302.

Svenonius, P. 2003. Limits on p: filling in holes vs. falling in holes. Nordlyd 2. 431-445.

Travis, L. 1984. Parameters and effects of word order variation. Unpublished doctoral dissertation. MIT.

Wunderlich, D. 1991. How do prepositional phrases fit into compositional syntax and semantics. Linguistics 29. 591-621.

Wunderlich, D. 2012. Lexical decomposition in grammar. In M. Werning, W. Hinzen \& E. Machery (eds.). Oxford handbook of compositionality. 307-327. Oxford University Press.

Zwarts, J. 1997. Vectors as relative positions: A compositional semantics of modified PPs. Fournal of Semantics 14. 57-86.

Zwarts, J. 2005. Prepositional aspect and the algebra of paths. Linguistics and Philosophy 28. 739-779.

Zwarts, J. \& Y. Winter. 2000. Vector space semantics: A model-theoretic analysis of locative prepositions. Journal of Logic, Language, and Information 9. 169-211. 


\section{A. Detailed Analyses}

For the detailed representation of überdachen and einlagern in this section, we use an extension of a basic DRT language (Kamp et al. 2011) with presuppositions and a $\lambda$ calculus for variable stores (Cooper 1983). $\lambda$-conversion selects the leftmost variable from the store. The storing of variables instead of immediate existantialization allows for a greater flexibility in the derivational process when it is necessary to distinguish between the introduction of existentially quantified discourse referents and manipulations of variables for discourse referents. A Discourse Representation Structure (DRS) $K$ with a presupposition $\mathrm{P}, \lambda$-abstracted variables $x, y$ and a store $v, z$ is represented as in (23). For more details on the semantic formalism, see (Roßdeutscher 2013b).

(23) $\lambda x \cdot \lambda y \cdot\langle\{P\}\langle v, z K\rangle\rangle$

The composition of DRSs is governed by applying $\lambda$-conversion and consequent merge of DRSs at each node of the syntactic structure. For example, the composition at the bottom of (27) on page 147 consists of a DRS taking a predicate (represented with capital letters) as an argument.

(24) $\lambda P\langle x, P(x)\rangle+\sqrt{ }$ dach $\rightarrow \lambda x \operatorname{roof}(x)$

Also, at each node in the composition it is checked whether presuppositions can be resolved by considering the new information made available. For example, when P' is merged with the DP introducing the direct object in (27), the presupposition $\{z\}$ introduced by the root $\sqrt{ }$ über is resolved to the discourse referent introduced with the direct object DP.

The introduction of discourse referents for states captures incorporation in that all conditions involving discourse referents predicated by the state are relocated into an inaccessible sub-DRS $K$ representing the semantic content of the state. For example, when $\mathrm{P}$ and SpaceP are merged with the predication of a state in (27), all conditions and existentializations involving discourse referents affected by the conceptual predicate APPL are grouped together in a new sub-DRS, thus rendering the nominal root $\sqrt{ }$ dach inaccessible as a discourse referent: 


$$
\langle x, \operatorname{roof}(x)]+\lambda u \cdot \lambda y \cdot \begin{aligned}
& s: A P P L(y, u) \\
& s: A y \cdot s: \begin{array}{l}
x \\
A P P L \\
\operatorname{roof}(x)
\end{array}
\end{aligned}
$$

In example (28), we use a version of Kratzer (1996)'s event identification principle applied to prepositional phrases in order to chain together the internal 'Ground' argument of a preposition and its figure. The referential argument to be identified is a set of vectors $v$ and the thematic role to be added is that of a Figure, see (26). Spatial refential arguments are existentialized at $\mathrm{pP}$.

(26) $\lambda x . \lambda v \cdot \operatorname{figure}(x, v)+\lambda v \cdot \begin{aligned} & u \\ & I N(u, v)\end{aligned} \rightarrow \lambda x \cdot \lambda v \cdot \begin{aligned} & u \\ & I N(u, v) \\ & \operatorname{figure}(x, v)\end{aligned}$ 
Measuring out the relation between formal and conceptual semantics

\section{A.1. überdachen}

(27) eine Terasse überdachen, full analysis

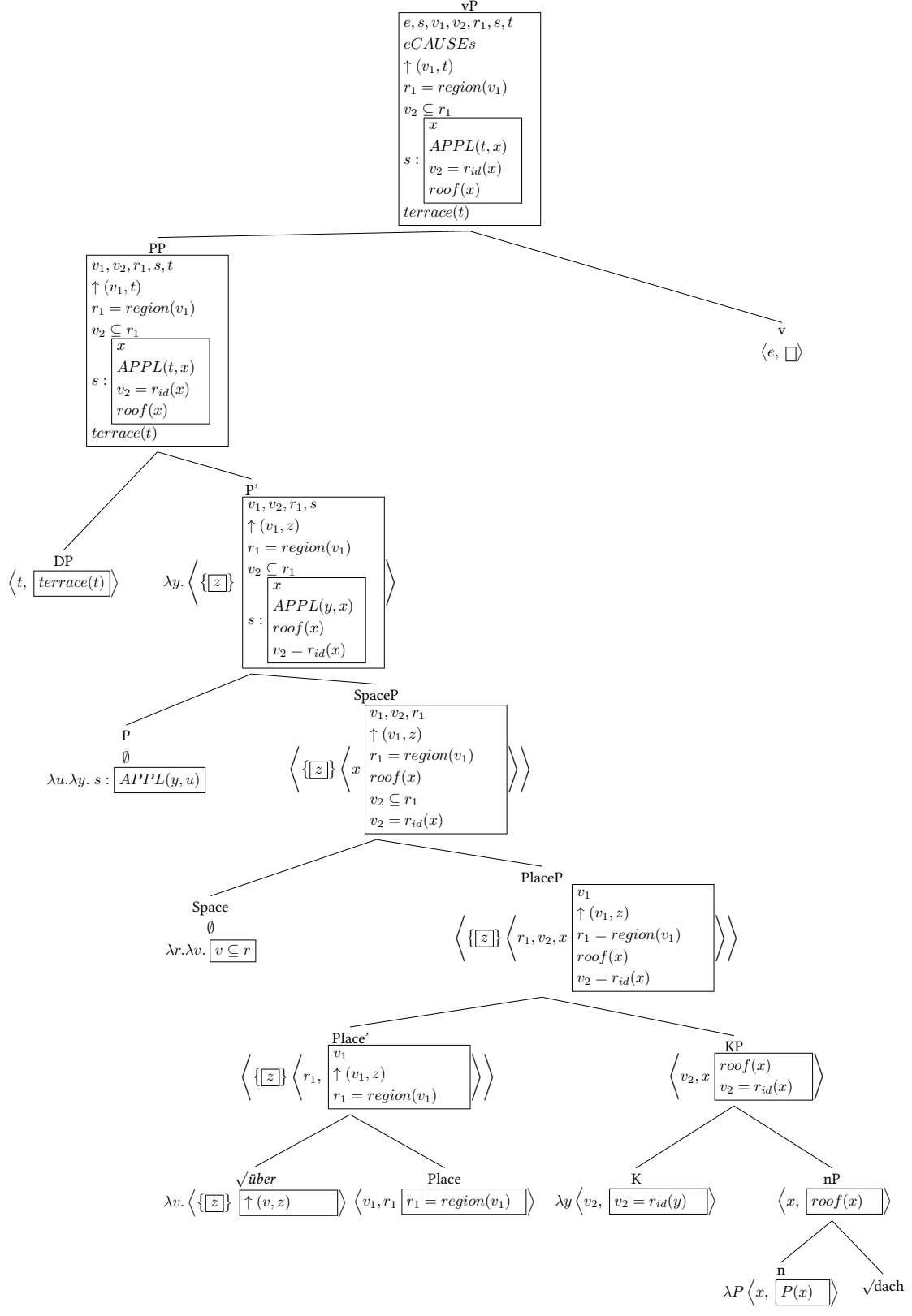




\section{A.2. einlagern}

(28) eine Flasche in den Keller ein(lagern), pP branch

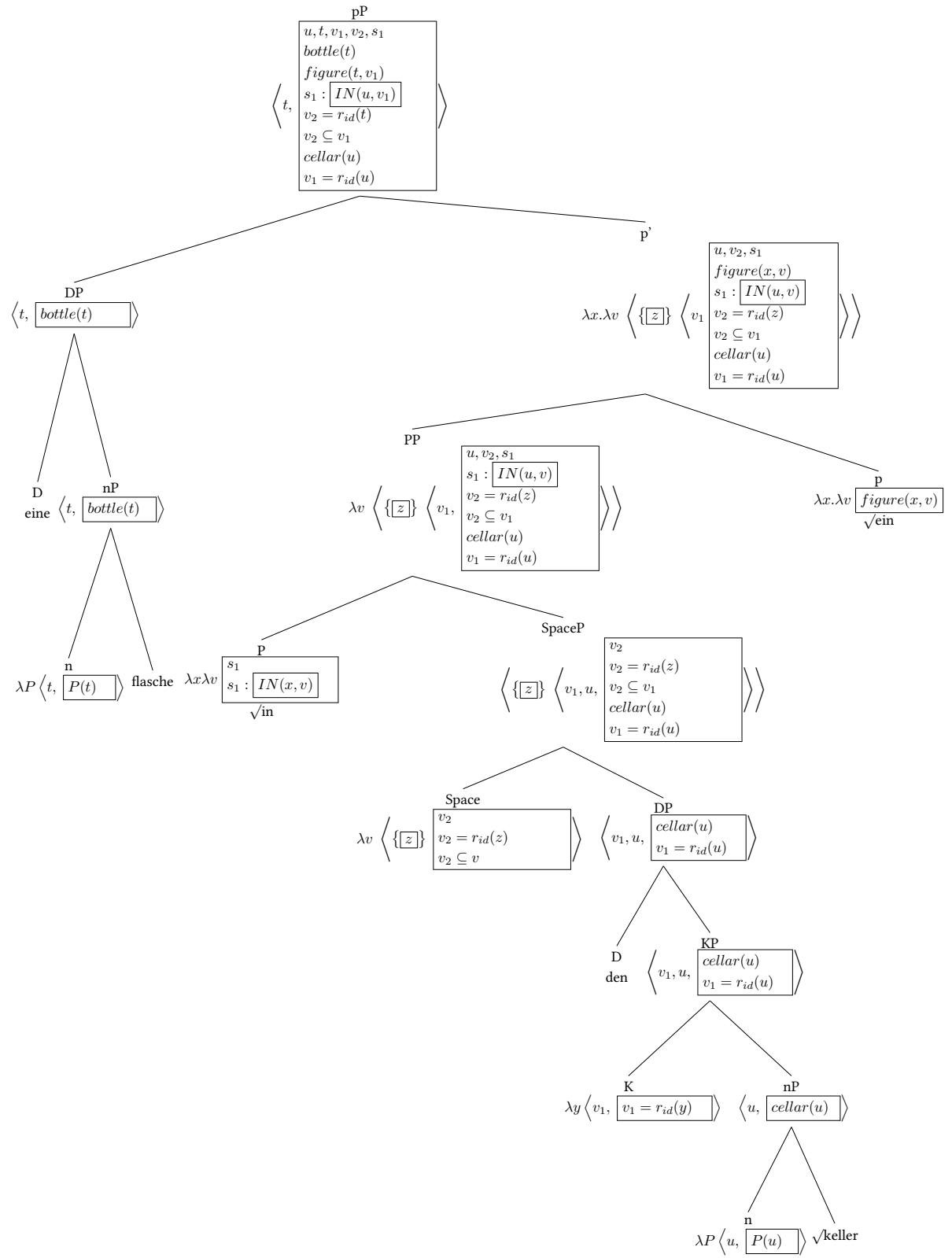


(29) (eine Flasche in den Keller ein)lagern, vP branch

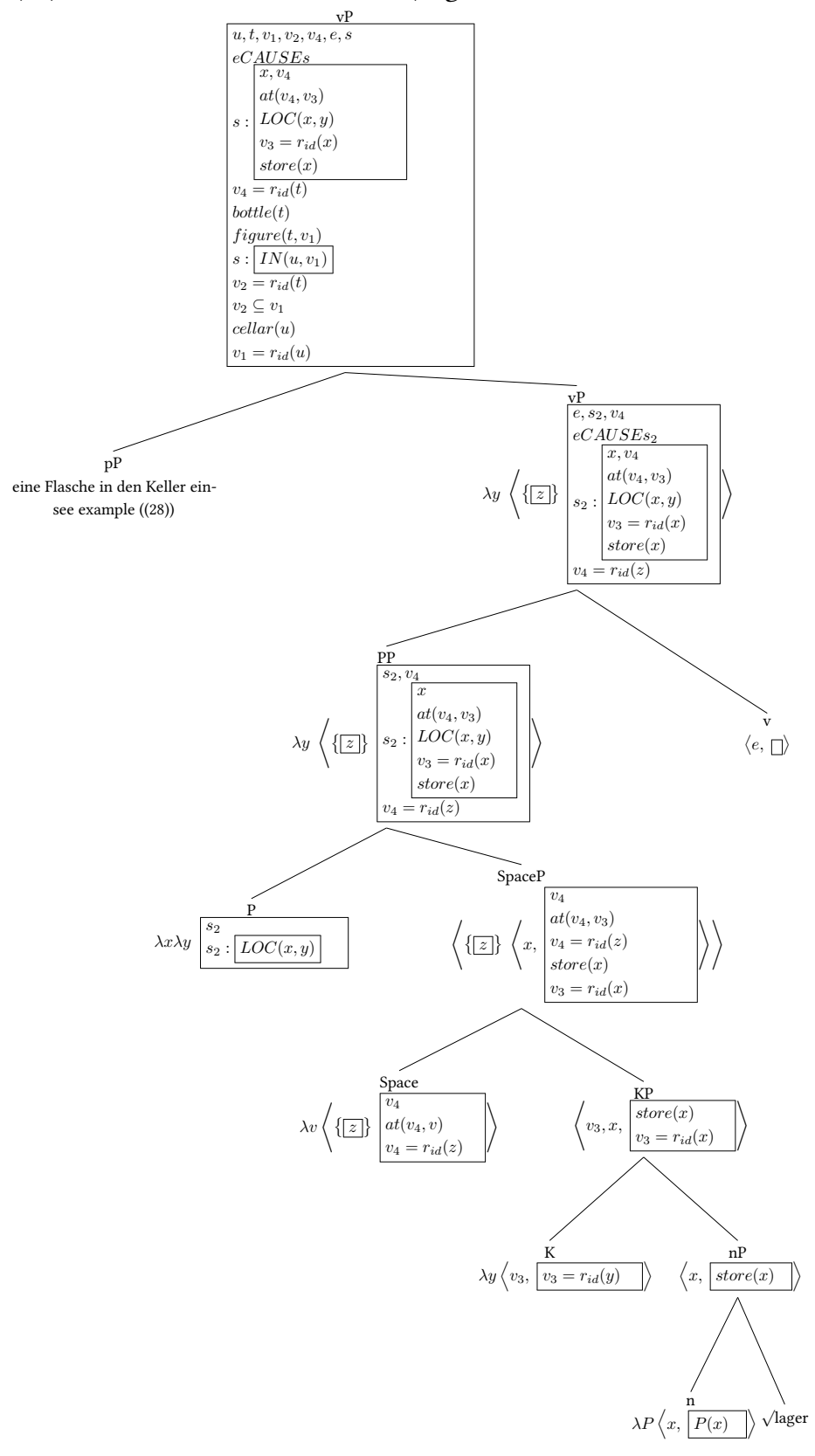




\section{Authors}

Tillmann Pross

IMS, Universität Stuttgart

tillmann.pross@ims.uni-stuttgart.de

Antje Roßdeutscher

IMS, Universität Stuttgart

antje@ims.uni-stuttgart.de 